\title{
Mistletoe fig (Ficus deltoidea Jack) leaf extract prevented postmenopausal osteoarthritis by attenuating inflammation and cartilage degradation in rat model
}

\begin{abstract}
Objective:

Ficus deltoidea Jack (mistletoe fig) is an ornamental plant found in various parts of the world and used as traditional herbal medicine in some countries. This study investigated the potential use of $\mathrm{F}$ deltoidea leaf extract to mitigate osteoarthritis (OA) in ovariectomized (estrogendeficient postmenopausal model) rats and the mechanisms involved. Diclofenac was used for comparison.
\end{abstract}

Methods:

Sprague-Dawley female rats (12 weeks old) were divided randomly into five groups $(\mathrm{n}=6)$ : healthy; nontreated OA; OA + diclofenac $(5 \mathrm{mg} / \mathrm{kg}) ; \mathrm{OA}+$ extract $(200 \mathrm{mg} / \mathrm{kg})$; and OA + extract $(400 \mathrm{mg} / \mathrm{kg})$. Two weeks after bilaterally ovariectomy, OA was induced by intraarticular injection of monosodium iodoacetate into the right knee joints. After 28 days of treatment, the rats were evaluated for knee OA via physical (radiological and histological observations), biochemical, enzyme-linked immunosorbent assay, and gene expression analysis, for inflammation and cartilage degradation biomarkers.

Results:

The osteoarthritic rats treated with the extract, and diclofenac showed significant reduction of cartilage erosion (via radiological, macroscopic, and histological images) compared with untreated osteoarthritic rats. The elevated serum interleukin- $1 \beta$, prostaglandin E2, and Ctelopeptide type II collagen levels in osteoarthritic rats were significantly reduced by $\mathrm{F}$ deltoidea leaf extract comparable to diclofenac. The extract significantly down-regulated the interleukin-1 $\beta$, prostaglandin E2 receptor, and matrix metalloproteinase-1 mRNA expressions in the osteoarthritic cartilages, similar to diclofenac.

\section{Conclusions:}

F deltoidea leaf extract mitigated postmenopausal osteoarthritic joint destruction by inhibiting inflammation and cartilage degradation enzymes, at an effective extract dose equivalent to about $60 \mathrm{mg} / \mathrm{kg}$ for humans. The main bioactive compounds are probably the antioxidative flavonoids vitexin and isovitexin.

Keyword: Articular cartilage; Ficus deltoidea; Inflammation; Osteoarthritis; Ovariectomy; Rat 